\title{
ALGUNOS FACTORES BIOMIECANICOS DE LA CARRERA EN ASCENSO
}

\section{Daniel Oliveros*}

\section{RESUMEN}

La carrera en ascenso es una forma de ejercitarse que se incluye en el entrenamiento de diferentes deportes y hace parte de clases de Educación Física. Algunos autores han estudiado esta manifestación desde diversos puntos de vista, en especial desde la fisiología. Este trabajo analiza la longitud y frecuencia de zancada en la carrera de ascenso y la compara con la carrera a nivel.

Además describe los cambios cinemáticos que ocurren en la actitud corporal al correr en ascenso.

El estudio se realizó con estudiantes de Educación Física (34 hombres y 25 mujeres). La metodología se basó en la medición de la longitud y frecuencia de zancada de 33 metros lanzados y en la filmación de las dos formas de correr. Los resultados muestran que la longitud de zancada se acorto durante la carrera en lomo con respecto a la carrera en nivel y que la frecuencia se aumenta en niveles de velocidad submaximales. Se aprecian cambios en los desplazamientos angulares en las acciones de brazos, piernas, tronco y cabeza. Los datos confirman lo encontrado por Nelson y Osterhoudt, aunque corresponden a velocidades e inclinaciones diferentes. Se complemento el trabajo con algunos datos fisiológicos y recomendaciones para la metodología del entrenamiento.

\section{INTRODUCCION}

La carrera en ascenso es un medio de entrenamiento difundido en el trabajo de diferentes deportes. Se encuentran referencias sobre atletismo, en particular 800 y 1500 (coe, 1983; Cock y showers, 1989 ), velocidad y heptatlón (Kersee, 1989), cross country (Hassard, 1989), fondo y semifondo (McDonell, 1989), saltos (Myers, 1986) y fútbol (Hernández, 1992; Bosco, 1992). Pero además, se reconoce que en la práctica, los entrenadores utilizan la carrera en ascenso con frecuencia y durante diferentes momentos de la periodización del entrenamiento. También, en la revisión bibliográfica se aprecian diferentes intenciones de desarrollo a través de esta modalidad de ejercicio: velocidad, potencia, resistencia orgánica, resistencia muscular, técnica de carrera y aumento de la frecuencia de zancada. En Colombia no se conoce ningún estudio al respecto.

Esta investigación del área de Biomecánica, se centró en las variaciones que sufren algunos parámetros cinemáticos de la carrera durante el ascenso. Los factores biomecánicos estudiados son la longitud y frecuencia de zancada, al igual que la posición de los diferentes sectores corporales (brazos, piernas, tronco, cabeza).

Aunque otros estudios se han concentrado en velocidades bajas o intermedias, el presente se focaliza en la carrera de velocidad.

De todas maneras, algunas implicaciones para la carrera de fondo en ascenso se pueden reconocer analógicamente. Participaron 35 hombres (peso promedio: $67.7 \mathrm{Kg}$.; estatura:

\footnotetext{
* Profesor Universidad Pedagógica Nacional 
$1.71 \mathrm{~m}$ ) y 25 mujeres (peso promedio: $54.5 \mathrm{Kg}$; estatura $1.62 \mathrm{~m}$ ), pertenecientes al departamento de educación física (E.F.) de la Universidad Pedagógica Nacional. El trabajo se desarrolló durante el segundo semestre de 1992 y el primero de 1993.

\section{¿POR QUÉ DEBE PROPENDER EL DEPARTAMENTO DE EDUCACIÓN FÍSICA EN LO ADMINISTRATIVO?}

En cuanto a los aspectos administrativos, los directivos del Departamento y de la Universidad deben ser facilita-dores del proceso de cualificación docente, auspiciando la programación constante de talleres y seminarios paras los profesores, otorgamiento de becas de posgrado, descarga académica para los maestros que se encuentran en proceso de cualificación, programación de cursos permanentes durante dos o tres veces a la semana, y la creación de rubros para la compra de libros. Pero, es la actitud del maestro en definitiva lo que impulsará o por el contrario frenará el desarrollo profesional, puesto que, todo maestro requiere de un continuo y permanente perfeccionamiento, sin el cual, permanecerá en una simple rutina que no sólo perjudicará su desarrollo personal y profesional, sino, el de todos aquellos que estén bajo su orientación. Mientras el maestro no comprenda que su labor debe trascender del sencillo proceso de Enseñanzaaprendizaje de una técnica o de un arte y que él es un intelectual de la cultura, sólo entonces es cuando se podrá lograr un avance profesional.

\section{FACTORES BIOMECANICOS DE LA CARRERA EN ASCENSO}

Durante la carrera en ascenso, se pueden distinguir principalmente dos superficies sobre las que se realizan las carreras: Ios planos inclinados (rampas, carreteras, lomas, etc.) y las escaleras, que pueden variar de altura y longitud de contrapaso y paso. Las anatomías se hacen sobre los planos inclinados y cuando sea necesario se hace la aclaración sobre las corridas en escalera.

\subsection{Trayectoria del Centro de Gravedad}

Durante la carrera a nivel, el desplazamiento del centro de gravedad (CG) se realiza en forma parabólica durante la fase de vuelo y con un leve descenso y ascenso en la correspondiente fase de apoyo. En la carretera de ascenso, la parábola de la trayectoria de CG es mas corta y mas ascendente, lo que provoca un acortamiento de la zancada. La necesidad de subir la trayectoria, coloca al cuerno en una posición mas erguida que en la carretera a nivel, aumentando el ángulo de despegue para la fase de vuelo.

\subsection{La Energía Cinética}

En cada zona de la carretera de ascenso hay una pérdida de energía cinética, que es superior a la de la carretera a nivel, por la disposición de la reacción del suelo con respecto al cuerpo del corredor. Esta relación al impacto del corredor sobre el suelo es mas horizontal y por lo tanto se opone mucho mas que en la carrera a nivel es decir, causa mas frenamiento horizontal que rebote vertical. 


\subsection{La Energía Potencial}

Debido a que el cuerpo del corredor debe ser ascendido a una nueva posición en cada zancada y que esta nueva localización corporal es mas elevada, la musculatura corporal debe realizar un trabajo adicional al aportado por la inercia, debido a las pérdidas de energía cinética. Se puede resaltar que el trabajo negativo de la amortiguación se realiza con menos flexión en la rodilla, y por la inclinación del plano, se debe realizar un poco mas de trabajo positivo en la fase propulsiva que en la carrera a nivel.

\subsection{Potencia}

La potencia mecánica que se realiza externamente durante la carrera en ascenso, tiene que ver con el peso del deportista, la velocidad de carrera y con la pendiente del plano inclinado que esté corriendo.

Si se considera que el trabajo es el producto de la fuerza desarrollada por la distancia recorrida por dicha fuerza, se puede relacionar el peso del corredor (W) y la altura ascendida $(\mathrm{H})$ así:

$$
\text { Trabajo }=W^{*} \mathbf{H}
$$

pero la altura puede relacionarse con la distancia y la inclinación del plano así:

$$
H=D * \operatorname{Sen} A,
$$

lo que implica que el trabajo resulta:

$$
\text { trabajo }=W * D * \operatorname{Sen} A
$$

Si se considera que la potencia es el trabajo realizado por unidad de tiempo, se tiene que:

$$
\text { Potencia }=(W * D * \operatorname{Sen} A) / T
$$

donde $T$ es el tiempo, y resulta entonces:

$$
\text { Potencia }={ }^{*} D * \text { Sen } A * D / T
$$

Ecuación que incluye D/T que es la velocidad V.

En resumen

$$
\text { Potencia }=\mathbf{W} * \operatorname{Sen} A * V \text {. }
$$

Si se examina cuidadosamente el factor Sen A para ángulos pequeños, se encuentra que el Sen A para ángulos de 1 a 10 grados es aproximadamente igual al ángulo expresado en porcentaje. Es decir, una pendiente de 3 grados es igual a una pendiente del tres por ciento $(3 \%)$. 


\subsection{Posición corporal y acciones articulares}

Como se estableció anteriormente, en la carrera (le ascenso se realiza más trabajo positivo para propulsar el cuerpo y es necesario ganar inercia nuevamente luego de la fase de apoyo, lo que exige que las acciones corporales sean mas amplias. Esto quiere decir que los brazos recorren un sector angular mayor, la rodilla delantera o de la pierna libre se eleva con más vigor y con mayor amplitud, aunque la pierna luego de perder contacto y flexionarse no lo hace con la misma amplitud que en la carrera a nivel. Puede destacarse que si la carrera se realiza en plano inclinado, el tendón de Aquiles se ve estirado por la dorsiflexión que forza el plano elevado de la punta del pie con respecto al talón. Esto no sucedería en la carrera en escaleras pues cada paso permite la colocación del pie en forma horizontal, sin afectar la longitud de los músculos posteriores de la pierna.

\section{ANÁLISIS Y DISCUSIÓN DE RESULTADOS}

La longitud de zancada se acortó de $2.16 \mathrm{~m}$ en plano hasta $1.67 \mathrm{~m}$ en ascenso para el grupo de hombres. Para las mujeres se redujo de $1.84 \mathrm{~m}$ a $1.41 \mathrm{~m}$. Es decir $0.49 \mathrm{~m}$ para hombres y $0.39 \mathrm{~m}$ para mujeres, en promedio. Por otro lado, la frecuencia disminuyó en el grupo femenino en 0.21 zanc/seg., pero para los hombres no se apreció el cambio. Es decir se conforma que la zancada se acorta cuando se corre en ascenso, pero aún no se puede afirmar lo mismo sobre la frecuencia, al parecer por la inmadurez de ritmo de carrera de los integrantes del grupo.

En cuanto a los cambios en posición y rango de movimiento articular, se aprecia que el tronco se mueve 6 grados en el plano, en tanto que en ascenso lo hace 11.5 grados. Esto implica mayor accionar de los músculos lumbares. La pierna de soporte recorre 57 grados en plano, mientras que en ascenso solo lo hace con 30 grados. Esto muestra que se disminuye el recorrido en 17 grados, valor muy parecido a los 18 grados de inclinación del plano. Los brazos realizan mas recorrido en ascenso, llevando el brazo adelantado 161 grados de recorrido en ascenso, en tanto que el plano solo se mueve 68 grados. Los muslos se elevan por encima de la horizontal 3 grados en ascenso, mientras que en plano le faltan 45 grados para la horizontal.

En el estudio de Nelson y Osterhoudt (1971), se usaron tres velocidades diferentes y tres pendientes. Las velocidades fueron $3.35,4.88$ y $6.40 \mathrm{~m} / \mathrm{s}$. El presente estudio se realizó a velocidad máxima para cada sujeto, resultando en promedio velocidades de $5.23 \mathrm{~m} / \mathrm{s}$ para mujeres y $7.05 \mathrm{~m} / \mathrm{s}$ para hombres. En este sentido se complementa el rango de velocidades estudiadas por los autores mencionados. Las pendientes fueron de 10 grados, $\mathrm{O}$ y 10 grados. Difieren con la usada en ascenso que fu\{e de 18 grados. En cuanto a la longitud de zancada se confirma la disminución de los valores para el ascenso. Para los valores de frecuencia, Nelson y Osterhoudt, confirman que la frecuencia aumenta en ascenso, aspecto que solo se observó en las mujeres. La diferencia podría explicarse si se considera que los hombres estaban en el máximo de velocidad de su carrera pero las mujeres sólo estaban en valores submáximos, es decir pueden mejorar mucho mas su frecuencia.

Grosser (1991), referencia aspectos fisiológicos, pero confirma que la carrera en ascenso implica cambios en los patrones de inervación muscular. Esto quiere decir que los recorridos articulares se aumentan durante el ascenso, provocando mayor actividad 
muscular. Esto confirma en parte los hallazgos del presente trabajo. De igual manera Gostill, señala que el gasto energético es superior en la carrera en ascenso(35\% mas). De esta afirmación se puede inferir que hay mayor gasto energético por que hay mayor actividad muscular y por tanto, los mayores recorridos articulares que se encontraron son aceptables.

\section{CONCLUSIONES}

El presente estudio confirmó que la longitud de zancada se acorta en la carrera en ascenso. Además, que la frecuencia puede aumentar par el ascenso, si no se encuentra la velocidad en valores maximales. Las acciones articulares que componen la estructura de movimiento de la carrera en ascenso, son de mayor amplitud que las carreras a nivel.

El método de medición de la longitud de zancada puede mejorarse en aspectos de precisión, pero el error manejado es pequeño. La metodología de copiar en acetato desde un televisor, aunque no ha sido estandarizada, representa una tecnología accesible a investigadores que no disponen de más. Estudios posteriores se necesitan para criticar su confiabilidad y precisión.

Los entrenadores interesados en incluir en sus prácticas la carrera en ascenso deben clarificar sus intenciones y limitar las repeticiones para evitar trabajos excesivos. McMahon (1984), demostró con trabajos de Margaria, que pendientes de $14 \%$ no permiten extender el trabajo mas allá de 2 minutos, y que descansos de 10 segundos resultan insuficientes, recomendado 30 segundos para trabajo de muchas repeticiones.

También, se podrá estudiar más sobre los efectos de la saltabilidad al correr en escaleras. Otro aspecto no contemplado en el presente estudio es la rotación del tronco en la carrera en ascenso, que se podría estudiar comparativamente en el plano y en el ascenso.

Se ha propuesto utilizar un test de potencia (Margaría P. Ej. ) para evaluar la potencia anaeróbica, pero también se podría examinar la potencia con un test de ascenso de 8 seg., especialmente para deportes de resistencia de velocidad que necesiten aceleraciones frecuentes o saltabilidad.

El los cálculos de potencia se puede apreciar que subir $1 \mathrm{~m}$ (test de Margaría) requiere de una potencia promedio de $75 \mathrm{Kg}$. / s y se realiza en aproximadamente $1 \mathrm{seg}$. En cambio, para subir $6 \mathrm{~m}$ se emplean $10 \mathrm{~s}$ y se requiere de una potencia de $45 \mathrm{Kg} . / \mathrm{s}$. De tal manera que con unos $8 \mathrm{~s}$. es posible mantener una demanda de potencia relativamente alta durante 8 veces más que en el primer caso. Si se aumenta la altura ascendida y por tanto la duración del ejercicio, la velocidad se reduce dramáticamente y la potencia desarrollada es reducida. Para una altura de $500 \mathrm{~m}$ (Como Monserrate) la potencia es apenas de 20 $\mathrm{Kg} . / \mathrm{s}$. debe notarse que para efectos de mejorar la técnica se debe usar distancias cortas ya que las largas reducen la amplitud de movimiento. Los trabajos en loma orientados al fondo tienen un impacto sobre el tendón de Aquiles que siempre debe ser considerado. 


\section{BIBLIOGRAFÍA}

1. BALLREICH, R. Model for estimating the influence of stride length and stride frecuency on the time in sprint events. En international series on biomechanics. Vol. IA - IB, University park Press: Baltimore, p. 208 - 212, 1976.

2. BOSCO, Carmelo. Aspectos fisiológicos de la preparación del futbolista. Paido Tribo: Barcelona, 1992.

3. COE, Peter. Trainning a world class $800 / 1500$ Athlete. En track and field quartely review, Vol. 83, No 3, Fall, 1983.

4. COSTILL, David. Inside Running. Bench Mark Press: Indianapolis, 1984.

5. ENOKA, Roger. Neuromechanical Basis of kinesiology. Human Kinetics: 1988.

6. GROSSER, HERMANN; TUSTER Y ZINTL: El movimiento deportivo. Bases anatómicas y biomecánicas. Edit. Martínez Roca: Barcelona, 1991.

7. HASSARD, Jerry. Cross Country contributions índex. En T.F.Q.R., Vol. 86, No. 4, Winter, 1986.

8. KERSEE, Bob. Philosophy of running training and methodology: Women sprinters and heptathlon. En T.F.Q.R.. Vol. 89, NO 1, Spring, 1989.

9. MCDONELL, Jhon. Arkansas Midlle distance and distance Trainning. En T.F.Q.R., Vol. 89, No 1, Spring, 1989.

10. MCMAHON, Thomas. Muscles, reflexe s and locomotion. Princeton University Press: Priceton, 1984.

11. MYERS, Larry. The jumping enets. En T.F.Q.R., Vol. 86, No 4, Winter, 1986.

12. NELSON; R Y OSTERHOUDT, R. Effects of altered slope and speed on the biomechanics of running. En Medicine and Sport. Vol. \&, Biomechanics II, p. 220-224. Karger y Bassel: 1971. 